\title{
Anomalous Origin of Coronary Artery from the Contralateral Aortic Sinus - Case Report
}

\author{
KAJAL KUMAR KARMOKER, KHANDAKER AISHA SIDDIKA, ARIF HOSSAIN, \\ MOHAMMAD ADIB AL JAVED, BIJOY DATTA
}

\author{
Department of cardiology, National Institute of Cardiovascular Disease (NICVD), Dhaka \\ Address of Correspondence: Dr Khandaker Aisha Siddika, Department of cardiology, NICVD, Dhaka, E-mail: \\ dr.aishajhagir.bsmmu@gmail.com
}

\begin{abstract}
Congenital coronary artery anomalies are rare heart defect that has been associated with myocardial ischemia and sudden death. Only 1-2\% of population having variation in the origin, course or distribution of the epicardial coronary arteries. Anomalous origin of coronary arteries may be present as isolated defect or as a part of complex congenital heart disease. The clinical presentation, diagnostic work up, prognosis and treatment of these anomalies are highly variable. Most of the patients are asymptomatic but manifestation of these patients are chest pain, dyspnoea, palpitation, dizziness, ventricular fibrillation, syncope and sudden death. It is the second most common cause of sudden death in young athletes. Selective coronary angiography is the gold standard for identification of such type of anomaly. Patients of anomalous origin of coronary artery from the opposite sinus may require medical treatment, coronary angioplasty with stenting or surgical repair.
\end{abstract}

Key words: Anomalous origin of coronary artery, Left coronary artery, Right coronary artery.

\section{Case Report (A):}

A 55 years old man, hypertensive, businessmen with positive family history of coronary artery disease admitted in National Institute of Cardiovascular Disease and Hospital with the complaints of chest pain on exertion for 2 years and syncopal attack for 2 times within last 1 month. His chest pain occurred even after 10-15 minutes of walking $\&$ relieved by taking rest. After $1^{\text {st }}$ syncopal attack he was admitted in khulna scote hospital and diagnosed as Complete heart block. Then treated by temporary Pacemaker, within 4 to 5 days patient revert to sinus rhythm and discharge from hospital. After 10 days patient again developed sycope and further treated by temporary pacemaker in NICVD. There was no history of shortness of breath. He was diagnosed as hypertensive for last 1 month and taking tablet amlodipin, tablet olmesartan and non diabetic. He was smoker and drug abuser for last 20 years.

His pulse rate was 26 per minute regular and high volume, all peripheral pulses were palpable and normal. Blood pressure was 140/60 mm $\mathrm{Hg}$ on lying flat. Precordium examination revealed no abnormality and both of lung bases were clear.

Among the investigation 12 lead electrocardiogram was showed complete heart block. Troponin I was eleveted, serum electrolytes, serum creatinine, blood sugar were normal. 2D M-mode and colour doppler echo cardiography was showed inferior wall hypokinesia with ejection fraction $50 \%$. Coronary anigiography revealed anomalous origin of right coronary artery (RCA) from left aortic sinus, dominant, good caliber vessel with 70$80 \%$ long segment stenosis in proximal part and $100 \%$ stenosis in mid part with retrograde filling from left coronary artery. Left main, left anterior descending coronary artery (LAD), left circumflex artery (LCX) were normal (Fig-1).

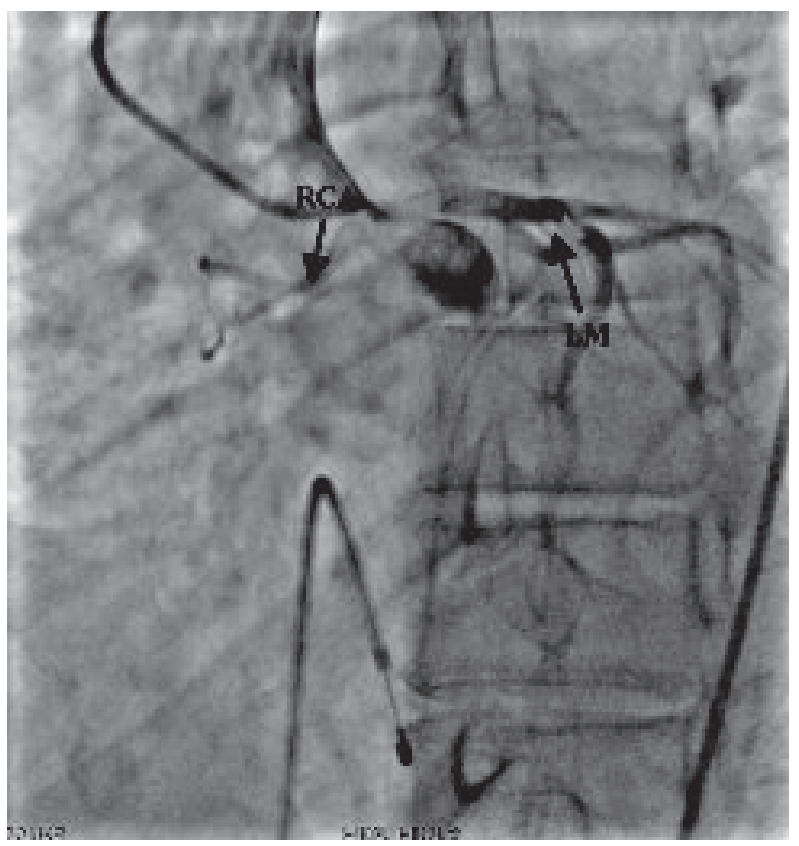

Fig.-I: Coronary angiogram showing RCA and LM both are originating from left aortic sinus. 


\section{Case Report (B):}

A 60 years old farmer admitted in NICVD complaining of chest pain for last 3 months. Chest pain initially occured on minimal exertion and even at rest. Then he was diagnosed as unstable angina and taking medical management, after that symptom improved. For last 15 days patient again developed resting chest pain that relieved by taking sub lingual nitroglycerin spray. There was no history of shortness of breath, palpitation or syncope. He was normotensive, non diabetic and smoker. He has positive family history of coronary artery disease.

On examination pulse rate was $80 / \mathrm{min}$ regular, normal volume, all the perpheral pulses were palpable and normal. Blood pressure was 130/80 mmHg on lying condition. JVP was normal and no leg oedema. Precordium examination reveals no abnormality and both of lung bases were clear.

12 lead electrocardiogram showed inferior ischemia ( $\mathrm{T}$ inversion in lead III, aVF). Troponin I, serum electrolytes, serum creatinine, blood sugar were normal. 2D M-mode and colour doppler echocardiography revealed normal study with good LV function. During coronary angiography at first we wanted to visualize the left coronary artery by left judkins catheter. But there was difficulties in catheter engagement. Then we tried to visualize the right coronary artery by right judkins catheter and which showed right coronary artery and left coronary artery both were originating from right aortic sinus. Right coronary artery (RCA) was dominant with $100 \%$ stenosis from distal part with retrograde filling from left circumflex artery. Left main was long and 50\% diseased. Left anterior descending coronary artery (LAD), LCX was good caliber vessels and diseases free (Fig-2).

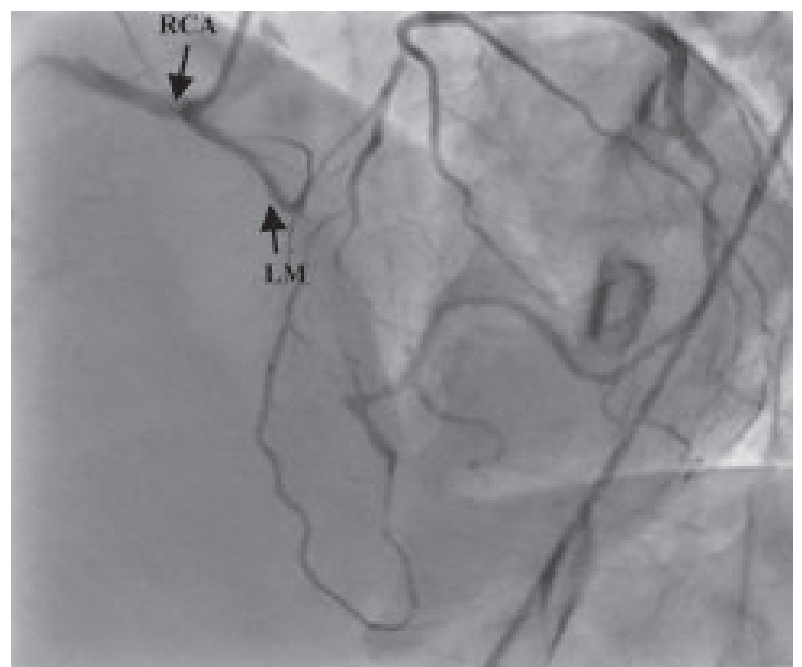

Fig.-2: Coronary angiogram showing origin of left main coronary artery and right coronary artery from right aortic sinus.

\section{Discussion:}

The incidence of anomalous origin of coronary artery is only $1-2 \%{ }^{1}$. It can be categorized as major or hemodynamically significant anomalies and minor ones. Classification of coronary anomalies are A. anomalies of origin and course B. Anomalies of intrinsic coronary artery anatomy C. anomalies of coronary termination D. Anomalous anastomotic vessels. Anomalies of origination and course may be I) Absent LM trunk II) Coronary ostium within the aortic root such as high and low take off III) Coronary ostium outside the normal coronary aortic sinuses such as pulmonary artery, ascending aorta, left ventrical, right ventrical. IV) Coronary ostium at wrong sinus such as RCA from left aortic sinus, left coronary artery from right aoritc sinus, LAD from right sinus, LCX from right sinus ${ }^{2}$.

The incidence of anomalous origination of right coronary artery from $\mathrm{Lt}$ sinus is $0.92 \%$ and left coronary artery from right sinus is $0.15 \% \%^{3}$. Right coronary artery from left sinus is 6 times more prevalent than left coronary artery. When left coronary artery arise from right sinus then left coronary artery follows 1 of 4 courses. These are i) between the aorta and pulmonary trunk, posteriorly and adjacent to the pulmonary trunk. ii) Passes anteriorly over the right ventricular outflow tract iii) Along the crista supraventricularis intramyocardially or subcendocardially, surfacing in the proximal intraventicular sulcus. iv. May arise from right of RCA and pass posteriorly to the aortic $\operatorname{root}^{3}$.

(Fig-III). The anomalous origin of RCA from left sinus generally courses between the aorta and pulmonary artery (fig-IV).

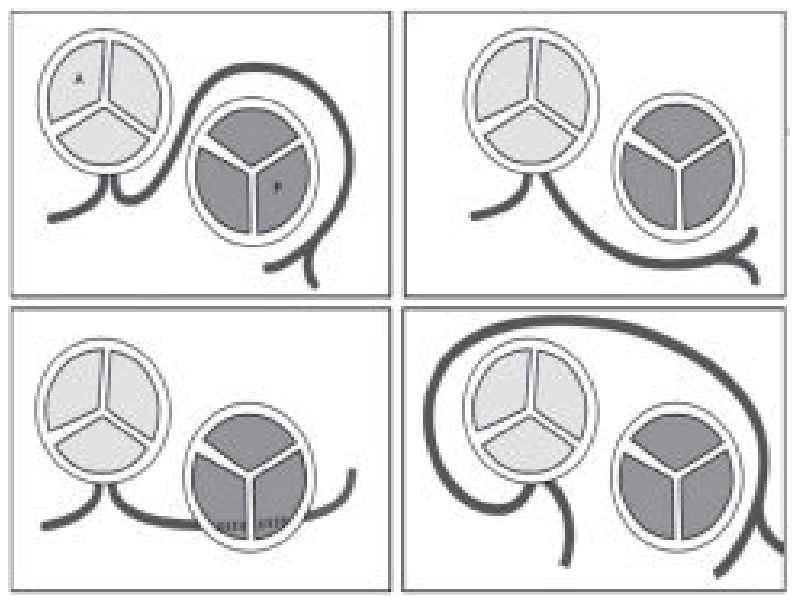

Fig.-3: Origin of the left main coronary artery (LMCA) from the right sinus of Valsalva. Top left: the LMCA passes between the aorta and pulmonary trunk. Top right: the LMCA passes anteriorly over the right ventricular outflow tract. Bottom left: the LMCA may course intramyocardially or subendocardially. Bottom right: the LMCA may rise to the right of the right coronary artery and pass posteriorly to the aortic root. 


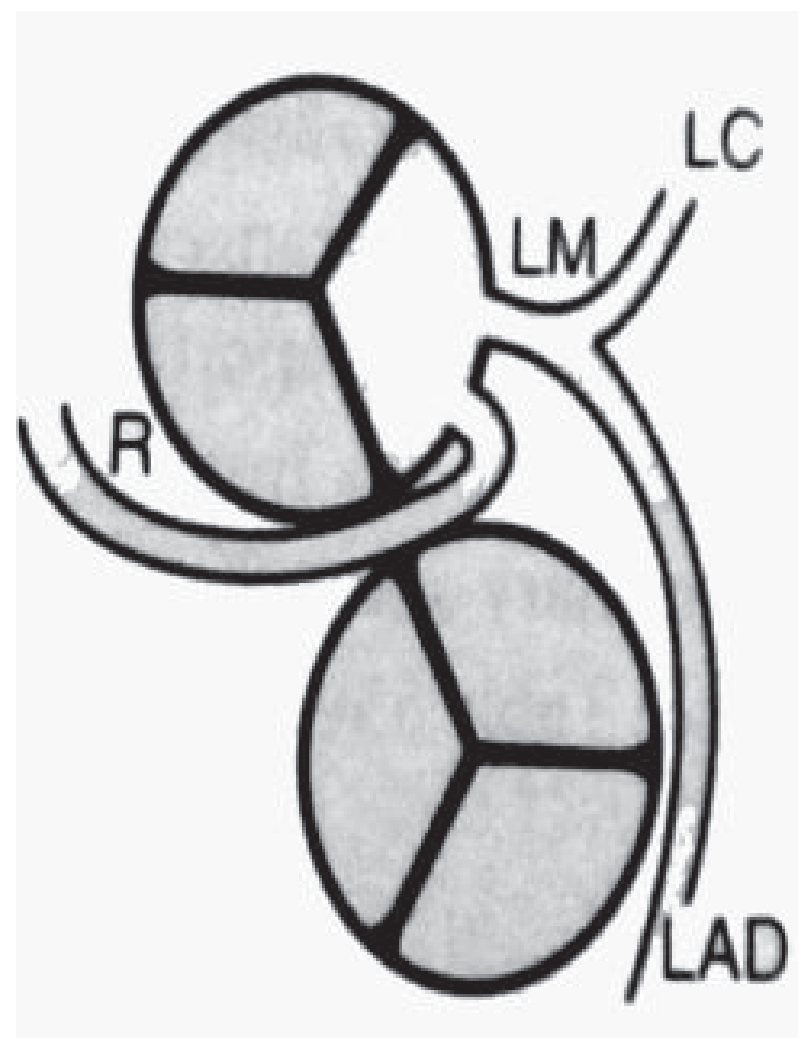

Fig.-4: Right and left main coronary artery arise from left aortic sinus.

Most patients of Anomalous origin of a coronary artery from the opposite sinus (ACAOS) are asymptomatic. Symptom typically occur in $2^{\text {nd }}$ to $4^{\text {th }}$ decade. Presentation of this patients are variable that including chest pain, dyspnoea, palpitation, dizziness, VF, syncope, sudden death. ${ }^{4}$ Sudden death usually associated with extreme exercise and occur in young adults. ${ }^{5}$ Whereas the other manifestations of ACAOS are more frequent for older adults and are related to onset of hypertension. The mechanism of ischemia, infarction and or sudden death in anomalous origin of a coronary artery from the opposite sinus (ACAOS) attributed to a number of factors, either singly or interrelated. These included slit like coronary orifice, acute angle of take-off, course between aorta and pulmonary artery, presence of intramural segments of coronary artery. ${ }^{5}$ Normal shape of coronary ostium is rounded or oval but in case of anomaly it is slit like in shape. When increase cardiac output, this aorta dilates with stretching of aortic wall, then slit-like ostium become severely narrowed. Compression of artery by aorta and pulmonary artery is unlikely in view of marked differences in diastolic pressure. ${ }^{1}$ Sudden death is seen only in young patients, the probable mechanism is progressive hardening of the aortic wall in adults. ${ }^{2}$ In case of anomalous coronary artery, increase risk of atherosclerosis. When RCA originate from left sinus $41.2 \%$ patients have RCA atherosclerosis ${ }^{7}$.

Patients suspected Anomalous origin of a coronary artery from the opposite sinus (ACAOS) require sequential investigation including electrocardiography, holter monitoring to document atrial or ventricular arrlythmias. Echocardiography, transthoracic and if needed transoesophageal. Echocardiography may identify the coronary origin and proximal course $\mathrm{e}^{4}$. CT coronary angiogram is confirm the diagnosis and identify true course of the vessels, angulations or kinking of the anomalous vessels and differentiating the benign from dangerous pathway. Cardiac MRI is also recommended. Nuclear stress testing is important to evaluate the effort induced ischemia and scars. Selective coronary angiography remains gold standard to rule out additional coronary artery atherosclerosis than to evaluate the severity of congenital obstruction at the proximal ectopic vessels. Selective coronary angiogram may be difficult, then aortic root injection or using Amplatz or multipurpose catheters is required. Intravascular ultrasonography (IVUS) is only needed for interventional management ${ }^{2}$.

Patients of ACAOS, who are symptomatic, have 3 treatment option. Medical treatment or observation, coronary angioplasty with stenting and surgical repair. Medical treatment with â blocker is probably as effective as restriction of strenious activity ${ }^{2,9}$. Showed that symptomatic patients become asymptomatic while treated with B-blockers in absence of luminal obstruction. Coronary angioplasty with stenting of obstructed proximal intramural segment of patients with right ACAOS is indicated when i) dysabling symptom and/or a high risk of sudden death. ii) More than 50\% stenosis with normal distal vessels on IVUS. iii) The supplied myocardial territory more than $1 / 3$ of total and iv) Reversible ischemia on nuclear stress test. Drug eluting stent is more preferable to avoid the restenosis. Surgical treatment is recommended for left-ACAOS irrespective of symptom².

There are significant differences in Anomalous left coronary artery (ALCA) and those with Anomalous Right coronary artery (ARCA) in respect to symptom, incidence, prognosis and treatment option. Sudden death is more common in patients of ALCA even in absence of symptoms. Right ACAOS is 6 times more common than then left ACAOS. The prognosis of ARCA is most often benign. Sudden death is extremely low in case of asymptomatic ARCA $^{6}$. 


\section{Conclusion:}

Anomalous coronary artery though rare but also present. Consequences of these anomalies are chest pain, arrhythmia, syncope and even sudden cardiac death. So anomalous coronaries should taken care off with great importance.

\section{References:}

1. Bruce, F, Waller. Nonatherosclerotic coronary heart disease. In: Fuster, V, Walsh, R \& Harringlon, RA (eds), Hurst's The Heart, 13 ${ }^{\text {th }}$ edn, McGraw Hill, New York, 2011; 1259-60.

2. Angelini, P, MD. Coronary artery anomalies an entity in search of an identity. Circulation. 2007; 115:1296-1305.

3. Hauser, M. Congenital anomalies of the coronary arteries. Heart 2005;91.1240-1245.
4. Angelini, P, Velasco, JA, Flamm, S. Coronary anomalies: incidence pathophysiology and clinical relevance. Circulation. 2002; 105:2449-54.

5. Liberthson RR. Nonatherosclerotic coronary artery disease. In: Eagle KA, ed. The practice of Cardiology. Boston: Little, Brown and Company; 1989:614-651.

6. Welton M, Gersony, MD, FACC. Management of anomalous coronary artery from the contralateral coronary sinus. Journal of the American College of Cardiology. 2007; 50:2083-4.

7. Yee, J, Arora, S, Lahewala, S, Kumar, V, Raizada, A, Anuwatworn, A, Gidwani, U, Sharma, S, Kini, A. Anomalous coronary arteries and its assocaition with incidence of coronary artery atherosclerosis: A study fom A tertiary care teaching hospital. JACC. 2016; 67:1224-161.

8. Hejmadi A, Sahn DJ. What is the most effective method of detecting anomalous coronary origin in symptomatic patients? $\mathrm{J} \mathrm{Am} \mathrm{Coli}$ Cardiol. 2003; 42:155-157.

9. Wann, S, M.D, Schuchard, G, M.D. Anomalous origin of the right coronary artery. $N$ Engl JMed. 2006;355:9. 